\title{
Evolutionary Computation for Optimal Ensemble Classifier in Lymphoma Cancer Classification*
}

\author{
Chanho Park and Sung-Bae Cho \\ Dept. of Computer Science, Yonsei University \\ 134 Shinchon-dong, Sudaemoon-ku, Seoul 120-749, Korea \\ cpark@candy.yonsei.ac.kr, sbcho@csai.yonsei.ac.kr
}

\begin{abstract}
Owing to the development of DNA microarray technologies, it is possible to get thousands of expression levels of genes at once. If we make the effective classification system with such acquired data, we can predict the class of new sample, whether it is normal or patient. For the classification system, we can use many feature selection methods and classifiers, but a method cannot be superior to the others absolutely for feature selection or classification. Ensemble classifier has been using to yield improved performance in this situation, but it is almost impossible to get all ensemble results, if there are many feature selection methods and classifiers to be used for ensemble. In this paper, we propose GA based method for searching optimal ensemble of feature-classifier pairs on Lymphoma cancer dataset. We have used two ensemble methods, and GA finds optimal ensemble very efficiently.
\end{abstract}

\section{Introduction}

Although early detection and correct class prediction of cancer have been seriously studied over the past years, there has been no exceptional way to work out this problem. It is because there can be so many pathways causing cancer, there exist tremendous number of varieties, and there are relatively small amount of experimental data. Recently, array technologies are developed enough to acquire thousands of gene expression levels at once. These gene expression profiles, however, are just simple sequence of numbers, so effective analysis tools are needed to find meaningful information in the numbers. Relating to this, many studies have been going on and published [1].

In general, the data from microarray is first normalized because raw data can be hardly used directly [2]. Then, we select informative genes by feature selection method, because the number of genes is much greater than that of samples, moreover many of them do not give help classification [3]. After feature selection, classifier operates with selected genes. Various feature selection methods and classifiers exist,

This paper was supported in part by Biometrics Engineering Research Center, KOSEF, and Brain Science and Engineering Research Program sponsored by Korean Ministry of Science and Technology in Korea. 
and they produce many feature-classifiers (combination of feature selection method and classifier). In this paper, we have used seven feature selection methods and six classifiers, leading to 42 different feature-classifiers. On the other hand, it is hard to find a feature-classifier that always shows better performance than any other ones. In this situation, we can expect to get wide and improved result through the ensemble classifier [4]. Ensemble is behaved with element feature-classifiers, and $2^{42}$ ensembles are available in our case. It is practically impossible to calculate all ensemble results, and if feature-classifiers are added, then the time increases exponentially.

To remedy these drawbacks, we propose a method based on the genetic algorithm for finding optimal ensemble of feature-classifier pairs efficiently. We have tried to test the proposed method on Lymphoma cancer dataset which is well known as benchmark data, and systematically analyze its usefulness.

The rest of the paper is organized as follows. In section 2, the backgrounds for this paper, such as DNA microarray and genetic algorithm, are illustrated. In section 3, the whole system for classifying gene expression profile is explained, such as feature selection methods, classifiers, ensemble classifier, and searching process by genetic algorithm. Section 4 presents the experiments and its results. Section 5 is the concluding remarks.

\section{Background}

\subsection{DNA Microarray}

DNA microarray consists of a large number of DNA molecules spotted in a systemic order on a solid substrate. Two DNA microarray types currently in wide use are cDNA array type and high density synthetic oligonucleotide array type. Here we only focus on oligonucleotide arrays. Oligonucleotide expression arrays include both short oligo (20-25mers) arrays and long oligo (50-70 mers) arrays [5].

DNA microarrays are composed of thousands of individual DNA sequences printed in a high density array on a glass microscope slide using a robotic arrayer. The relative abundance of these spotted DNA sequences in two DNA or RNA samples may be assessed by monitoring the differential hybridization of the two samples to the sequences on the array. For mRNA samples, the two samples are reverse-transcribed into cDNA, labeled using different fluorescent dyes mixed (red-fluorescent dye Cy5 and green-fluorescent dye Cy3). After the hybridization of these samples with the arrayed DNA probes, the slides are imaged using scanner that makes fluorescence measurements for each dye. The log ratio between the two intensities of each dye is used as the gene expression data.

Hybridization of fluorescently labeled RNA and DNA-derived samples to DNA microarrays allows the monitoring of gene expression or occurrence of polymorphisms in genomic DNA.

$$
\text { gene_expression }=\log _{2} \frac{\operatorname{Int}(\mathrm{Cy} 5)}{\operatorname{Int}(\mathrm{Cy} 3)}
$$




\subsection{Genetic Algorithm}

Genetic algorithms are stochastic search methods that have been successfully applied in many search, optimization, and machine learning problems [6]. Unlike most other optimization techniques, GAs maintain a population of encoded tentative solutions that are competitively manipulated by applying some variation operators to find a global optimum. A population consists of many chromosomes that can be a candidate solution. A chromosome is composed of bit strings that express a specific status or value.

A sequential GA proceeds in an iterative manner by generating new populations of strings from the old ones. Every string is the encoded (binary, real, ...) version of a candidate solution. An evaluation function associates a fitness measure to every string indicating its fitness to the problem. The standard GA applies genetic operators such as selection, crossover, and mutation on an initially random population in order to compute a whole generation of new strings.

\section{Cancer Prediction System}

The classification architecture for DNA microarray is composed of a series of processes to classify samples. The architecture contains feature selection, classifier, and finding optimal ensemble using GA. The system is as shown in Fig. 1.

\subsection{Gene Selection Methods}

We use seven feature selection methods to select informative genes. They are based on representative three fields of feature selection, such as statistical correlation, measurement of distance, and information theory.

Statistical approach. Using the statistical correlation analysis, we can see the linear relationship and the direction of relation between two variables. Correlation coefficient $r$ varies from -1 to +1 , so that the data distributed near the line biased to (+) direction will have positive coefficients, and the data near the line biased to (-) direction will have negative coefficients.

Suppose that we have a gene expression pattern $g_{i}(i=1 \sim m$, where $m$ is the number of genes). Each $g_{i}$ is a vector of gene expression levels from $n$ samples, $g_{i}=$ $\left(e_{1}, e_{2}, \ldots, e_{\mathrm{n}}\right)$. Some elements are examples of class 1 , and the others are those from class 0 . An ideal gene pattern that belongs to class 1 is defined by $g_{\text {ideal_cl }}=(1, \ldots, 1$, $0, \ldots, 0)$, so that all the elements from class 1 's samples are 1 and the others are 0 .

In this paper, we have calculated the correlation coefficients between this $g_{\text {ideal }}$ and the expression pattern of each gene. When we have two vectors $X$ and $Y$ that contain $N$ elements, $r_{\text {Pearson }}(\mathrm{PC})$ and $r_{\text {Spearman }}(\mathrm{SC})$ are calculated as follows: 


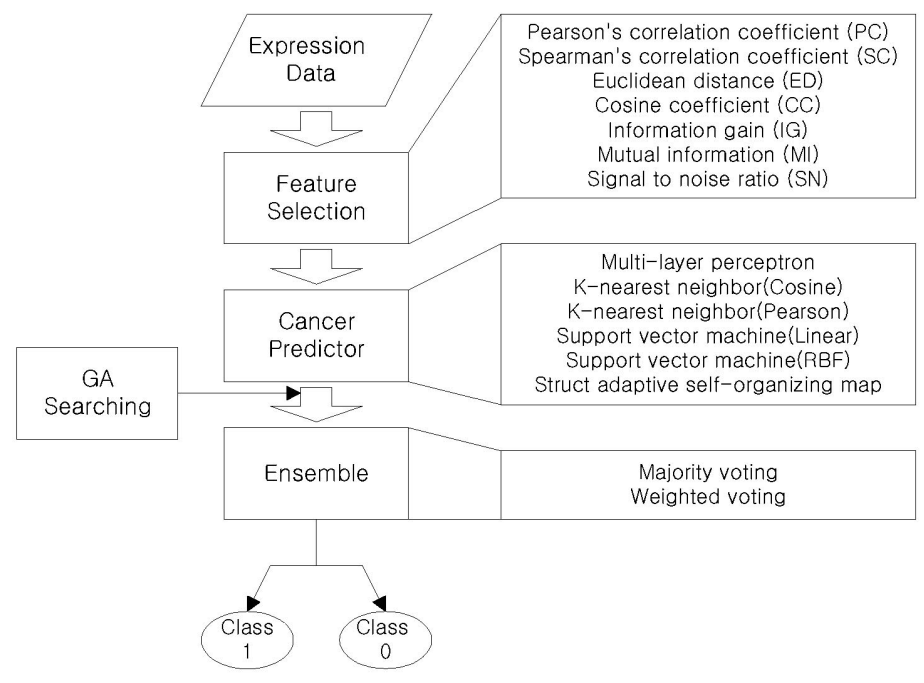

Fig. 1. The Cancer Prediction System

$$
\begin{gathered}
r_{\text {Pearson }}=\frac{\sum X Y-\frac{\sum X \sum Y}{N}}{\sqrt{\left(\sum X^{2}-\frac{\left(\sum X\right)^{2}}{N}\right)\left(\sum Y^{2}-\frac{\left.\left(\sum Y\right)^{2}\right)}{N}\right)}} \\
r_{\text {Spearman }}=1-\frac{6 \sum\left(D_{x}-D_{y}\right)^{2}}{N\left(N^{2}-1\right)}
\end{gathered}
$$

where $D_{x}$ and $D_{y}$ are the rank matrices of $\boldsymbol{X}$ and $\boldsymbol{Y}$, respectively.

Distance measure approach. The similarity between two input vectors $\boldsymbol{X}$ and $\boldsymbol{Y}$ can be thought of as distance. Distance is a measure on how far the two vectors are located, and the distance between $g_{\text {ideal } \_l}$ and $g_{i}$ tells us how much the $g_{i}$ is likely to the class 1. Calculating the distance between them, if it is bigger than certain threshold, the gene $g_{i}$ would belong to class 1 , otherwise $g_{i}$ belongs to class 0 . In this paper, we have adopted Euclidean distance $\left(r_{\text {Eclidean }}, \mathrm{ED}\right)$ and cosine coefficient $\left(r_{\text {Cosine }}, \mathrm{CC}\right)$ represented by the following equations:

$$
\begin{aligned}
& r_{\text {Eclidean }}=\sqrt{\sum(X-Y)^{2}} \\
& r_{\text {Cosine }}=\frac{\sum X Y}{\sqrt{\sum X^{2} \sum Y^{2}}}
\end{aligned}
$$

Information-theoretic approach. We have utilized the information gain and mutual information that are widely used in many fields such as text categorization and data mining. If we count the number of genes excited $\left(P\left(g_{i}\right)\right)$ or not excited $\left(P\left(g_{i}\right)\right)$ in 
category $c_{j}\left(P\left(c_{j}\right)\right)$, the coefficients of the information gain (IG) and mutual information (MI) become as follows:

$$
\begin{gathered}
I G\left(g_{i}, c_{j}\right)=P\left(g_{i} \mid c_{j}\right) \log \frac{P\left(g_{i} \mid c_{j}\right)}{P\left(c_{j}\right) \cdot P\left(g_{i}\right)}+P\left(\bar{g}_{i} \mid c_{i}\right) \log \frac{P\left(\bar{g}_{i} \mid c_{j}\right)}{P\left(c_{j}\right) \cdot P\left(\bar{g}_{i}\right)} \\
M I\left(g_{i}, c_{j}\right)=\log \frac{P\left(g_{i}, c_{j}\right)}{P\left(c_{j}\right) \cdot P\left(g_{i}\right)}
\end{gathered}
$$

Mutual information tells us the dependency relationship between two probabilistic variables of events. If two events are completely independent, the mutual information is 0 . The more they are related, the higher the mutual information gets. Information gain is used when the features of samples are extracted by inducing the relationship between gene and class by the presence frequency of the gene in the sample. Information gain measures the goodness of gene using the presence and absence within the corresponding class.

For each gene $g_{i}$, some are from class 1 , and some are from class 0 . If we calculate the mean $\mu$ and standard deviation $\sigma$ from the distribution of gene expressions within their classes, the signal to noise ratio of gene $g_{i}, S N\left(g_{i}\right)$, is defined by:

$$
S N\left(g_{i}\right)=\frac{\mu_{c 1}\left(g_{i}\right)-\mu_{c 0}\left(g_{i}\right)}{\sigma_{c 1}\left(g_{i}\right)+\sigma_{c 0}\left(g_{i}\right)}
$$

\subsection{Classifiers}

Multilayer perceptron. Error backpropagation neural network is a feed-forward multilayer perceptron (MLP) that is applied in many fields due to its powerful and stable learning algorithm [7]. The neural network learns the training examples by adjusting the synaptic weight of neurons according to the error occurred on the output layer. The power of the backpropagation algorithm lies in two main aspects: local for updating the synaptic weights and biases, and efficient for computing all the partial derivatives of the cost function with respect to these free parameters.

K-nearest neighbor. $K$-nearest neighbor $(\mathrm{KNN})$ is one of the most common methods for memory based induction. Given an input vector, KNN extracts $k$ closest vectors in the reference set based on similarity measures, and makes decision for the label of input vector using the labels of the $k$ nearest neighbors [8]. Pearson's correlation and cosine coefficient have been used as the similarity measure.

Support vector machine. Support vector machine (SVM) estimates the function classifying the data into two classes [9]. SVM builds up a hyperplane as the decision surface in such a way to maximize the margin of separation between positive and negative examples. SVM achieves this by the structural risk minimization principle that the error rate of a learning machine on the test data is bounded by the sum of the training-error rate and a term that depends on the Vapnik-Chervonenkis (VC) dimension. We have used linear kernel and RBF kernel in SVM ${ }^{\text {light }}$ module. (available at: http://svmlight.joachims.org/) 
Structure adaptive SOM. Even though SOM is well known for its good performance of topology preserving, it is difficult to apply it to practical classification since the topology should be fixed before training. A structure adaptive self-organizing map (SASOM) was proposed to overcome this shortcoming [10]. SASOM starts with $4 \times 4$ map, and dynamically splits the output nodes of the map, where the data from different classes are mixed, trained with the LVQ learning algorithm.

\subsection{Ensemble Classifier}

Though there are various feature selection methods and classifiers, it is hard to find the perfect method for classification. Besides it is difficult to decide the number of informative genes, and setting of parameters. In a situation like these, we cannot expect that one feature-classifier always produces good performance. At this time, ensemble classifier can be used usefully through the combination of feature-classifier pairs. Researchers are used as ensemble methods such as majority voting, Bayesian average, neural network, and so on [11].

If we just use a feature-classifier pair, we get only 42 results of classification because we have used seven feature selection methods and six classifiers. However, we can get about 4-tera results by ensemble classifier. It means that we can search much wider solution space. We have applied this idea to the classification framework as shown in Fig. 1. We have chosen majority voting and weighted voting as ensemble methods, they are shown in the Table 1.

Table 1. Ensemble methods (when $x$ is input, $c_{1 i}(x)=1$, if $e_{i}(x)=1$, otherwise $c_{1 i}(x)=$ $0, c_{0 i}(x)=1$, if $e_{i}(x)=0$, otherwise $c_{0 i}(x)=0, w_{i}$ is accuracy of $e_{i}(x)$, and $e_{i}(x)$ is element feature-classifier)

\begin{tabular}{ccc}
\hline Ensemble method & Output & Condition \\
\hline \multirow{2}{*}{ Majority voting } & 1 & $\sum_{i}\left(c_{1 i}(x)\right)>\sum_{i}\left(c_{0 i}(x)\right)$ \\
\cline { 2 - 3 } & 0 & otherwise \\
\hline \multirow{2}{*}{ Weighted voting } & 1 & $\sum_{i}\left(c_{1 i}(x) w_{i}\right)>\sum_{i}\left(c_{0 i}(x) w_{i}\right)$ \\
\cline { 2 - 3 } & 0 & otherwise \\
\hline
\end{tabular}

\subsection{Searching Optimal Ensemble Using GA}

It takes so long time to calculate all possible ensembles. Moreover, if we added one feature-classifier pair, it would increase spending time exponentially. Therefore efficient method for finding optimal ensemble is needed, so that we have proposed the method with GA. The structure of chromosome is as shown in Fig. 2. 


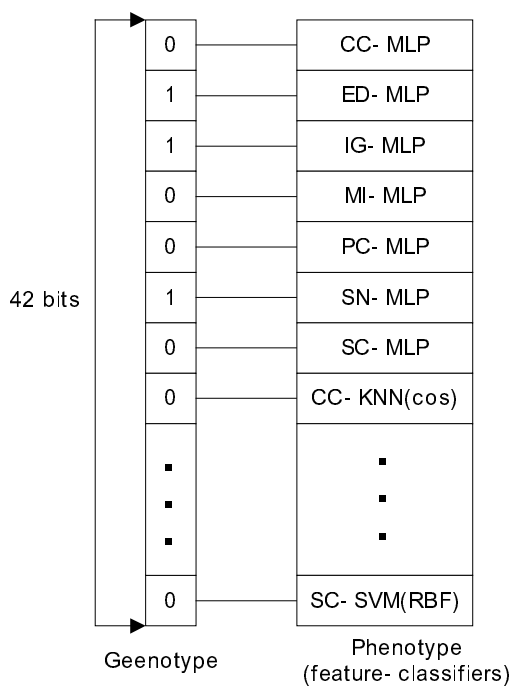

Fig. 2. The Design of Chromosome

Each chromosome is composed of 42 bits, and each bit means that the corresponding feature-classifier is included or not. Each bit corresponding specific feature-classifier like Fig. 2. Fig. 2 means the ensemble of second, third, and sixth feature-classifier pairs because those bits are 1. After initial population is created, fitness is evaluated with ensemble result.

\section{Experiments}

\subsection{Experimental Dataset}

B cell diffuse large cell lymphoma(B-DLCL) is a heterogeneous group of tumors, based on significant variations in morphology, clinical presentation, and response to treatment. Gene expression profiling has revealed two distinct tumor subtypes of BDLCL: germinal center B cell-like DLCL and activated B cell-like DLCL [12]. Lymphoma dataset consists of 24 samples of GC B-like and 23 samples of activated B-like. 22 out of 47 samples were used as training data and the remaining were used as test data in this paper. (Available at: http://genome-www.stanford.edu/lymphoma)

\subsection{Experimental Environment}

The experimental process is composed of gene selection, classification, and searching optimal ensemble using GA. For gene selection, we rank the genes according to its feature score, and select 25 highly scored genes. For classifier, we have used MLP 
with 8 hidden nodes, 2 output nodes, $0.01 \sim 0.50$ of learning rate, 0.9 of momentum, 500 of maximum iterations, and $98 \%$ of goal accuracy. In $k \mathrm{NN}$, we have set $k$ from 1 to 8 , and used Pearson correlation coefficient and cosine coefficient as similarity measures. We have used SVM with linear and RBF kernel function. In SASOM, we have used initial $4 \times 4$ map which has rectangle shape.

Preliminary experiments for searching optimal ensemble using GA show converge to local minimum when we use only tens of chromosomes. So we set the sizes of population to $100,200,500,1000,1500$, and 2000. Many crossover rates and mutation rates are applied to various size of population. We use roulette wheel rule as a selection method, and give higher fitness to chromosome that have small number of feature-classifier pairs.

\subsection{Results of Element Feature-Classifier}

There are results for all element feature-classifier pairs in Table 2. $K \mathrm{NN}(\mathrm{Cosine})$ classifier records good accuracy and IG shows outstanding performance for all classifiers. Feature selection methods based on information-theoretic approach show better performance than others in this dataset.

Table 2. Recognition rate with features and classifiers (\%) in Lymphoma dataset

\begin{tabular}{ccccccc}
\hline & \multirow{2}{*}{ MLP } & \multirow{2}{*}{ SASOM } & \multicolumn{2}{c}{ SVM } & \multicolumn{2}{c}{ KNN } \\
\cline { 4 - 7 } & & & Linear & RBF & Cosine & Pearson \\
\hline PC & 64.0 & 48.0 & 56.0 & 60.0 & 76.0 & 60.0 \\
SC & 60.0 & 68.0 & 44.0 & 44.0 & 60.0 & 60.0 \\
ED & 56.0 & 52.0 & 56.0 & 56.0 & 68.0 & 56.0 \\
CC & 68.0 & 52.0 & 56.0 & 56.0 & 72.0 & 60.0 \\
IG & 92.0 & 84.0 & 92.0 & 92.0 & 92.0 & 92.0 \\
MI & 72.0 & 64.0 & 64.0 & 64.0 & 64.0 & 80.0 \\
SN & 76.0 & 76.0 & 72.0 & 76.0 & 80.0 & 76.0 \\
\hline Avg. & 69.7 & 63.4 & 62.9 & 63.4 & 73.1 & 69.1 \\
\hline
\end{tabular}

\subsection{Results of Optimal Ensemble with GA}

Before finding optimal ensemble, we investigate the change of average fitness to see if GA evolves. It is because optimal ensemble can be found accidentally. Fig. 3 shows the change of average fitness. We can see that average fitness increases by iteration and it converge after about 100 iterations. Fig. 4 is a plot for relation of population size and generation that find optimal ensemble. It shows that population size is inverse proportion to the finding generation.

We could not get good performances by element feature-classifier pairs. It means that there exists high possibility of improving performance, and GA practically finds ensembles that record improved performance than any other element feature-classifier pairs. The accuracy of element feature-classifier pair is $44 \sim 92 \%$, and average is $67 \%$. 
Nevertheless, GA finds the ensemble of $100 \%$ that is composed of supplemented pairs of feature-classifier. Ensembles searched by GA have ranged 92 100\% accuracy, and recorded $96.67 \%$ on average. Some optimal ensembles are in Table 3.

The real usefulness of GA lies in its time-efficiency. We have experimented with all the ensembles that are composed of 3,5 and 7 feature-classifier pairs without using GA, and it takes about 20 minutes. Therefore, it is almost impossible to test all the ensembles. However, it takes only 60 seconds averagely with GA of 2000 population size when finding optimal ensemble.

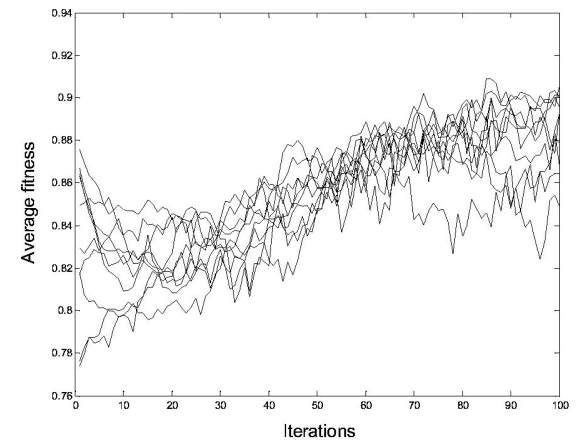

Fig. 3. The Change of Average Fitness

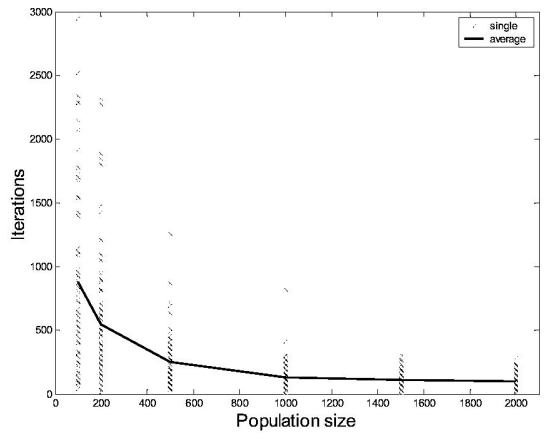

Fig. 4. The Population Size and Generation that finds Optimal Ensemble

Table 3. Optimal ensembles of Lymphoma dataset

\begin{tabular}{|c|c|c|}
\hline Methods & Feature-classifier pairs & \\
\hline \multirow[t]{2}{*}{ Majority voting } & $\begin{array}{l}\text { CC-KNN(P), MI-KNN(C), SN-KNN(C), } \\
\text { SVM(L) }\end{array}$ & SS-SASOM, IG- \\
\hline & $\begin{array}{l}\text { IG-KNN(C), MI-KNN(C), PC-KNN(C), } \\
\text { SASOM }\end{array}$ & $\mathrm{SN}-\mathrm{KNN}(\mathrm{P}), \mathrm{SN}-$ \\
\hline \multirow[t]{2}{*}{$\begin{array}{l}\text { Weighted } \\
\text { voting }\end{array}$} & $\begin{array}{l}\text { IG-KNN(C), IG-KNN(P), PC-KNN(P), } \\
\text { SASOM }\end{array}$ & $\mathrm{SN}-\mathrm{KNN}(\mathrm{P}), \quad \mathrm{CC}-$ \\
\hline & $\begin{array}{l}\text { IG-KNN(C), MI-KNN(C), SN-KNN(C), } \\
\text { SASOM, IG-SASOM, PC-SVM(R) }\end{array}$ & $\mathrm{SN}-\mathrm{KNN}(\mathrm{P}), \quad \mathrm{CC}-$ \\
\hline
\end{tabular}

\section{Concluding Remarks}

This paper uses GA for efficiently analyzing gene expression profiles. Experimental results show that GA finds ensembles that produce higher performance than any individual feature-classifier pairs. Though we can find optimal ensemble without using GA, it is so time-consuming work for evaluating all ensembles. GA finds 
optimal solution quickly, and it is more useful when feature selection method or classifier is added to the system. In addition, we can see that GA finds optimal solution through the increasing average fitness. This means that chromosomes evolve to right way.

On the other hand, though we have just used simple GA and ensemble methods, we will investigate more methods and conditions. Moreover, we are going to experiment on the other datasets to establish our proposed method. Though we cannot know the meaning of genes that related optimal ensembles found by GA, but there exists biological worth, and these studies will be good for interdisciplinary work.

\section{References}

1. Golub, T. R., et al.: Molecular classification of cancer: Class discovery and class prediction by gene expression monitoring. In Science, vol. 286, pp. 531-537, 1999.

2. Yang, Y. H., Dudoit, S., Luu, P. and Speed, T. P.: Normalization for cDNA microarray data. In SPIE Bios 2001, San Jose, California, pp. 1-12, 2001.

3. Model, F., et al.: Feature selection for DNA methylation based cancer classification. In Bioinformatics, vol. 17 Suppl. 1, pp. s157-s164, 2001.

4. Cho, S.-B. and Ryu, J.-W.: Classifying gene expression data of cancer using classifier ensemble with mutually exclusive features. In Proc. of the IEEE, vol. 90, no. 11, pp. 1744$1753,2002$.

5. Li, F. and Stormo, G. D.: Selection of optimal DNA oligos for gene expression arrays. In Bioinformatics. vol. 17, no. 11, pp. 1067-1076, 2001.

6. Mitchell, T. M.: Machine Learning, Carnegie Mellon University, 1997.

7. Khan, J., et al.: Classification and diagnostic prediction of cancers using gene expression profiling and artificial neural networks. In Nature, vol. 7, no. 6, pp. 673-679, 2001.

8. Duda, R. O., Hart, P. E., and Stork, D. G.: Pattern Classification, 2nd Ed. Wiley Interscience, 2001.

9. Furey, T. S., et al.: Support vector machine classification and validation of cancer tissue samples using microarray expression data. In Bioinformatics, vol. 16, no. 10, pp. 906-914, 2000.

10. Kim, H. D. and Cho, S.-B.: Genetic optimization of structure-adaptive self-organizing map for efficient classification. In Proc. of International Conference on Soft Computing, pp. 34-39, 2000.

11. Ben-Dor, A., et al.: Tissue classification with gene expression profiles. In Journal of Computational Biology, vol. 7. pp. 559-584, 2000.

12. Alizadeh, A. A., et al.: Distinct types of diffuse large B-cell lymphoma identified by gene expression profiling. In Nature, vol. 403, pp. 503-511, 2000. 